\title{
Remote Patient Management in Peritoneal Dialysis: Opportunities and Challenges
}

\author{
Shailesh Agarwal • Martin Wilkie
}

Sheffield Kidney Institute, Sheffield Teaching Hospital NHS Foundation Trust, Sheffield, UK

\begin{abstract}
Telehealth describes the use of information and communication technologies to deliver healthcare remotely. Given the twin healthcare pressures of demographic change, where increased numbers of people suffer long-term conditions and there is requirement to deliver care ever more cost effectively, there is a considerable need to make the best use of these technologies in the redesign of clinical services. The opportunity for telehealth in peritoneal dialysis (PD) is to strengthen an already enabled population of resilient individuals who undertake their treatment in the community while at the same time providing the opportunity for more people to have their treatment at home. Relevant to this discussion are the challenges that characterize PD. In many parts of the world, uptake is low, technique survival is disappointing, and access to the therapy is impacted by a range of factors such as socioeconomic status, ethnic minority status, and social isolation. There are barriers to learning the technique that may be physical, educational or cognitive, and support by a family member or paid carer may be required. Can remote monitoring impact these factors - and would its widespread use build confidence in patient perceptions that would enable more people to benefit from home dialysis? For that to happen, a clear sighted goal is required for the development of high quality evidence that examines the opportunities and challenges around its use, and understands the needs of all stakeholders. This is key for appropriate and patient-centered implementation that benefits healthcare outcomes, which matter to patients and to those who provide healthcare. This will not happen by accident and must be carefully built into the plan for the roll out and evaluation of these technologies.
\end{abstract}




\section{Background}

The idea of remote patient management (RPM) was highlighted in the public consciousness in June 2014 when Apple Inc introduced a partnership with the Mayo Clinic to allow health data collected from the Apple Watch to be seamlessly transmitted to the organization. The stated goal was "a connected health strategy that puts patients at the center of healthcare technology." A baffling range of digital technologies are able to remotely monitor patient health activity including smartphones/personal digital assistants, wearables, biosensors, computerized systems or multiple components of these. The conditions in which these technologies have received most attention are chronic diseases such as asthma, heart failure, and diabetes [1]. A systematic meta-review concluded that while telehealth-mediated self-management was not consistently superior to usual care, none of the reviews reported negative effects [2]. A specific example of positive impact comes from a randomized controlled trial of inflammatory bowel disease management in the Netherlands where patients were assigned to care either via a telemedicine system (myIBDcoach) that monitors and registers disease activity or standard care. Its use was safe and associated with reduced outpatient visits and hospital admissions compared with standard care, as well as high patient-reported quality of care, thus providing evidence of value to healthcare providers, systems, and patients [3].

Relevant to this discussion is the evidence that among people with long-term conditions, those who have greater involvement in their own care are likely to have better outcomes. Patients who are more "activated" are those who have "the knowledge, skills and confidence in managing their own health and healthcare" [4]. Those with greater levels of patient activation are better able to make effective use of healthcare, whereas lower activation associates with more frequent emergency room attendance and greater likelihood to be readmitted following discharge from hospital. Taking steps to enhance activation involves sharing information with patients in a manner that best informs and educates them, and provides the basis for shared decision making, and this has been prioritized in guidance from the National Institute of Health and Care Excellence in England on Renal Replacement Therapy [5]. Unfortunately, UK kidney patients rate their experience of sharing decisions about their care below all other patient reported measures [6], and therefore there is much to be done to make this a reality for patients. An important question is to what extent better connected health systems, as demonstrated through telemedicine, can strengthen patient involvement in their own care, and through this mechanism improve outcomes.

Remote monitoring has many potential attractions for people who dialyze in the community and some of these were highlighted as part of a kidney health 
initiative on advancing technologies [7]. Pilot experience of its use to support patients at home on peritoneal dialysis (PD) has been reported from several countries. One consideration is whether this "virtual" supervision may help to reduce a common concern of healthcare teams about less frequent oversight for patients on PD that may contribute to its underutilization. Increased remote support with embedded educational content that reinforces best practices has the potential to lead to higher patient satisfaction, better outcomes, and potentially better acceptance of PD as a dialysis modality [8]. A survey from a home dialysis program in North America reported that most patients were interested in participating in telemedicine and thought that it would lead to a reduction in the requirement for clinic and emergency room visits [9]. For healthcare professionals, clearer data gives better information to inform patient management decisions. However, it is important to recognize the collaborative healthcare model of supported self-care, upon which home dialysis is based. Patients are trained to be competent and develop a level of independence and trust which is important not to undermine through intrusive supervision. There are 3 main stakeholders in the RPM debate: patients, healthcare professionals such as doctors and nurses, and provider organizations. In this chapter, we briefly explore the most important opportunities and challenges relevant to these groups. It is essential that the patient voice is at the heart of development, otherwise the technology will lack relevance to their needs.

\section{Opportunities and Challenges}

Telehealth encompasses a broad range of possible functions such as remotely monitoring biometric parameters, direct 2 -way communication with the patient and their carer, adjusting prescriptions, and educational functions $[7,8,10]$. In other words, it allows the creation of a "virtual clinic" where many aspects of patient care can be conducted without the requirement for the patient to attend physically, or indeed for the healthcare team to visit the patient at home. With each of these functions come opportunities (e.g., closer monitoring, more timely management decisions, strengthened education), but by the same token attendant challenges (how best to rationalize the information, the most effective use of data to support decision making, designing education to suit individual circumstances, ensuring that the patient continues to feel supported) (Fig. 1). As with every technology, the goal is to maximize benefit while being cognizant of drawbacks and the most appropriate ways to minimize these. There are a number of domains that can be potentially impacted by these approaches and these are summarized in Table 1 . 
Table 1. Opportunities and challenges for remote monitoring in PD

\begin{tabular}{|c|c|c|}
\hline & Opportunities & Challenges \\
\hline Patient & $\begin{array}{l}\text { More responsive support - } \\
\text { troubleshooting of problems } \\
\text { Reduced patient anxiety } \\
\text { More accurate and easier record } \\
\text { keeping } \\
\text { Opportunities to enhance education, } \\
\text { health literacy, and patient activation } \\
\text { Reduced sense of isolation and } \\
\text { improved patient satisfaction } \\
\text { Conversations with clinical teams } \\
\text { more useful as necessary data are } \\
\text { available } \\
\text { Less travel to the hospital }\end{array}$ & $\begin{array}{l}\text { Potentially intrusive } \\
\text { Concerns regarding technological } \\
\text { aspects including data security } \\
\text { Worries about reduced staff } \\
\text { contact } \\
\text { Does not cover all potentially } \\
\text { helpful domains - for example, no } \\
\text { information on effluent cell counts }\end{array}$ \\
\hline $\begin{array}{l}\text { Healthcare } \\
\text { provider }\end{array}$ & $\begin{array}{l}\text { Ability to provide more responsive } \\
\text { care - for example, quicker resolution } \\
\text { of catheter flow problems } \\
\text { Remote adjustment of device settings } \\
\text { Easier longitudinal evaluation of } \\
\text { clinical data, for example, } \\
\text { ultrafiltration volumes, weights, and } \\
\text { blood pressure } \\
\text { Reduced requirement for home visits } \\
\text { Reduced frequency of clinic visits in } \\
\text { selected patients through the use of } \\
\text { nurse-led remote clinics } \\
\text { Reduced hospitalization } \\
\text { Better outcomes } \\
\text { Improved healthcare delivery - } \\
\text { greater efficiency, cost effectiveness, } \\
\text { safety } \\
\text { Monitoring of supply use to ensure } \\
\text { timely delivery of stock }\end{array}$ & $\begin{array}{l}\text { Acceptance of technology } \\
\text { Responding to the technological } \\
\text { challenge - requires initial } \\
\text { investment in time to learn and } \\
\text { implement the technology } \\
\text { Scheduling appropriate data } \\
\text { review processes - with } \\
\text { appropriate guidance for setting of } \\
\text { flags and moving from reactive to } \\
\text { proactive strategies } \\
\text { Decisions about which data to } \\
\text { monitor and the best way to do } \\
\text { this to ensure effective use of } \\
\text { healthcare time while avoiding } \\
\text { data fatigue } \\
\text { Liability associated with delayed } \\
\text { review of significant flags }\end{array}$ \\
\hline $\begin{array}{l}\text { Healthcare } \\
\text { system }\end{array}$ & $\begin{array}{l}\text { Greater uptake of home therapies } \\
\text { Reduced transportation costs - } \\
\text { ecological benefits }\end{array}$ & $\begin{array}{l}\text { Providing evidence of value } \\
\text { Technology failure - for example, } \\
\text { lost transmission of data due to } \\
\text { poor signal coverage } \\
\text { Absorbing higher costs of the } \\
\text { technology }\end{array}$ \\
\hline
\end{tabular}

$\mathrm{PD}$, peritoneal dialysis.

\section{Implementing Remote Monitoring of PD}

Remote monitoring of peritoneal dialysis (RMPD) represents a technological development that has considerable potential to improve care in a range of ways. There are several key phases to consider when planning the implementation of 
Fig. 1. How does telehealth contribute to the continuum of supported self-care?

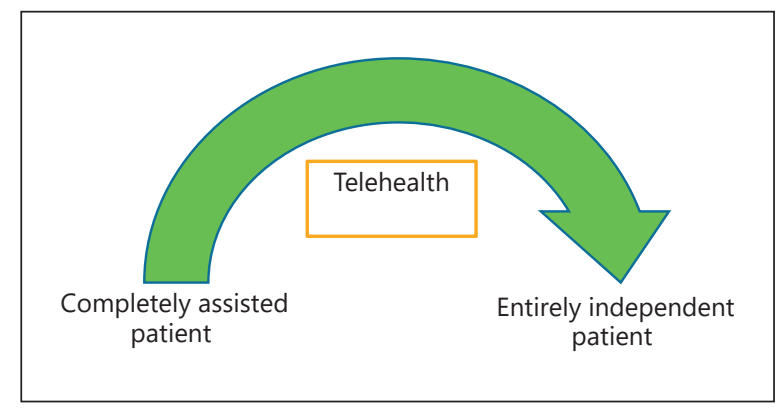

such a program. These include ensuring organizational support; detailed planning, consultation with a wide range of stakeholders and procurement; piloting and review; and finally moving to full-scale adoption. At the outset, the team will need to agree which patients it is best to involve first. It would be logical to start with those who live furthest from the centre, are more difficult to schedule visits with or present a higher risk for a range of reasons. Alternatively, it may be used for all automated peritoneal dialysis (APD) patients at a particular center, while recognizing that RMPD technology incorporated as part of APD machines is not available for those using manual continuous ambulatory peritoneal dialysis (CAPD).

Clinical teams will need to delegate responsibility for reviewing online biometric data and plan a schedule so that it forms a part of the regular working timetable. It is necessary to agree the setting of alerts so that attention is drawn to areas of highest priority such as catheter flow issues, ultrafiltration (UF) volumes, weight and BP changes, as well as missed treatment sessions, whilst minimizing the risk of data fatigue. Patients should have access to their own data, be educated in its relevance, and involved in its review. The biometric and cycler performance data requires to be included in patient progress reviews and multidisciplinary meetings. The adoption of RMPD should be subject to careful evaluation (e.g., auditing the data review procedures) to ensure that the hoped for goals are obtained, that burdens and risks are minimized, and that the experience is positive for patients.

\section{Patient Independence and Increased PD Uptake}

PD places a significant burden of self-care on patients, and this can be a daunting prospect. Patient education and support are central to successful self-care. Alongside ensuring appropriate techniques are learned to minimize complications from PD, patients also need to develop an awareness of when to seek help. This requires regular retraining as well as easy and prompt access to clinical support from a dedicated team who can identify complications and act responsive- 
ly. In a 2009 survey about factors behind patient decisions on renal replacement therapy modality, lack of supervision was the most commonly cited reason for avoiding home therapies such as PD [11], whereas patients who do not get their chosen modality cite lack of confidence and insufficient education among the reasons [12]. Physical and cognitive difficulties limit the ability to train to perform PD. On the contrary, carers experience fatigue that can impact the success of the therapy. By providing an increased level of support, remote monitoring may be able to give more people the confidence to take on aspects of their own care. Remote biometric monitoring can reduce the feeling of isolation for individuals as they record and act on information, providing an enhanced "apprenticeship" beyond the end of the formal training period.

\section{Reducing Technique Failure}

Technique failure (TF) is a major concern for PD as a therapy and a worry for individuals. It can pose tough challenges such as the requirement to be constantly vigilant to signs of peritoneal infection [13]. Reflecting its importance, TF has been adopted as the primary outcome measure for the International PD Outcomes and Practice Patterns Study [14]. In the first year of therapy, catheterrelated problems are the most important cause [15] and it is here that RM may provide an opportunity for prompt identification of drainage problems triggering appropriate investigations and interventions before it becomes a problem. In an illustrative case report of a patient who required urgent start APD, red flag dashboard alerts displayed on the Sharesource interface indicated prolonged drain times leading to clinical evaluation and subsequent surgical repositioning for catheter displacement. After that, drain times returned to normal as indicated by the disappearance of flag alerts and unremarkable cycle volume profiles [16]. In this way, early identification can prevent the patient becoming demoralized and sleep deprived due to recurrent night machine alarms, as well as experiencing reduced solute and volume removal.

Figure 2a presents an example of cycler profiles reviewed remotely in a patient from our practice who was commenced on APD using a tidal prescription, demonstrating impaired catheter function that was resolved after attending to bowel function (Fig. 2b).

\section{Review of Biometric Data}

A pilot study of remote biometric monitoring of 269 patients on PD demonstrated the feasibility of its use since uptake was reported in $90 \%$ and there was no association with gender, educational, socioeconomic or employment status, or baseline experience with computers. Remote biometric monitoring was associated with adherence to self-monitoring of BP and weight. It allowed for in- 


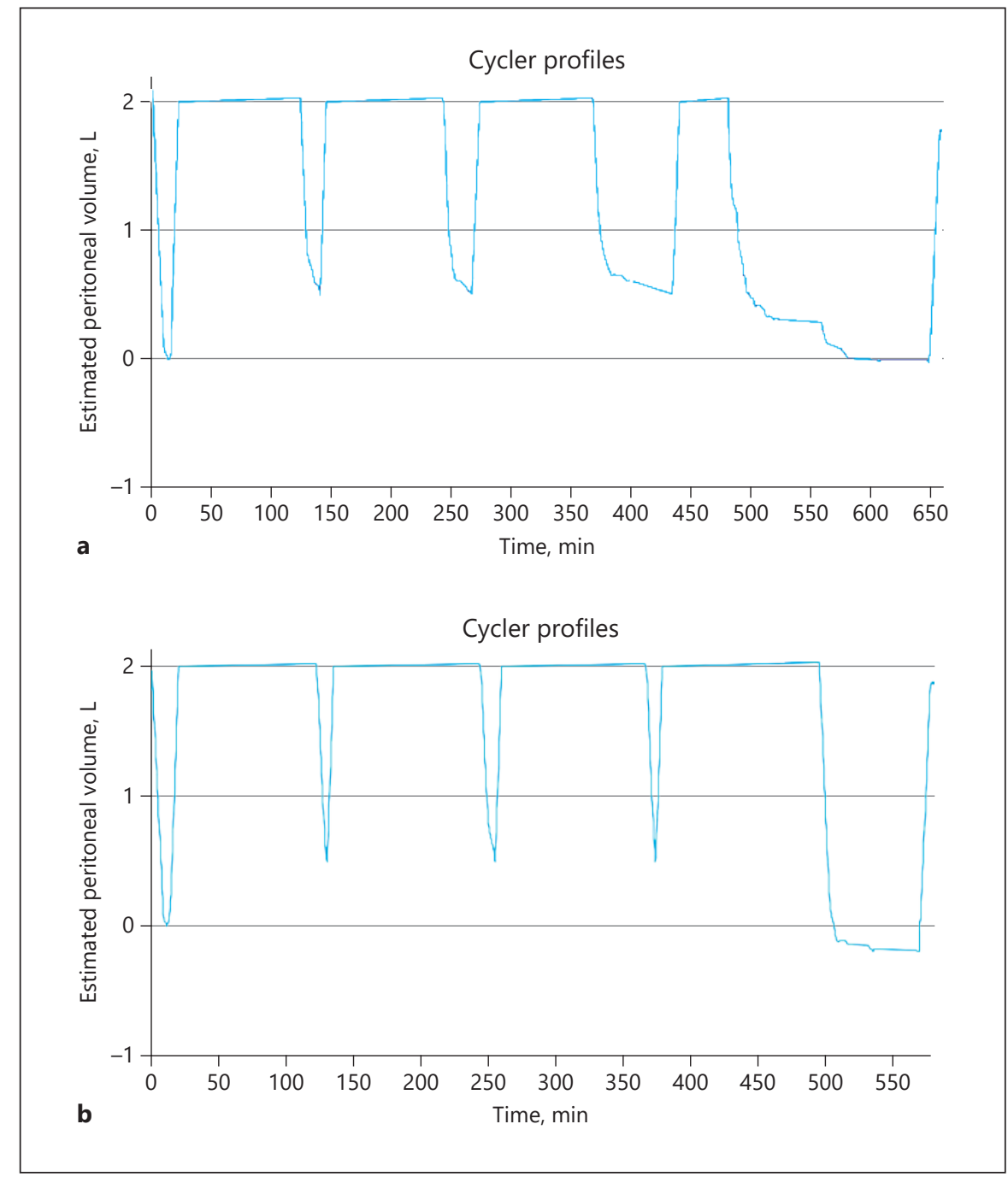

Fig. 2. a Cycler profile demonstrating impaired catheter drainage. b Cycler profile following resolution of catheter drainage.

creased communication between patients and PD clinical staff based on realtime patient data that enabled providers to potentially improve adherence and outcomes [17]. Changes in UF volumes, weight, and blood pressures are best understood as a trend over several days - with comparisons over the course of a month being particularly useful. Figure 3 shows a sudden change in UF demonstrated on review of sequential Sharesource data that was likely to have indicated a mechanical problem. Plain radiology confirmed the catheter to be ap- 


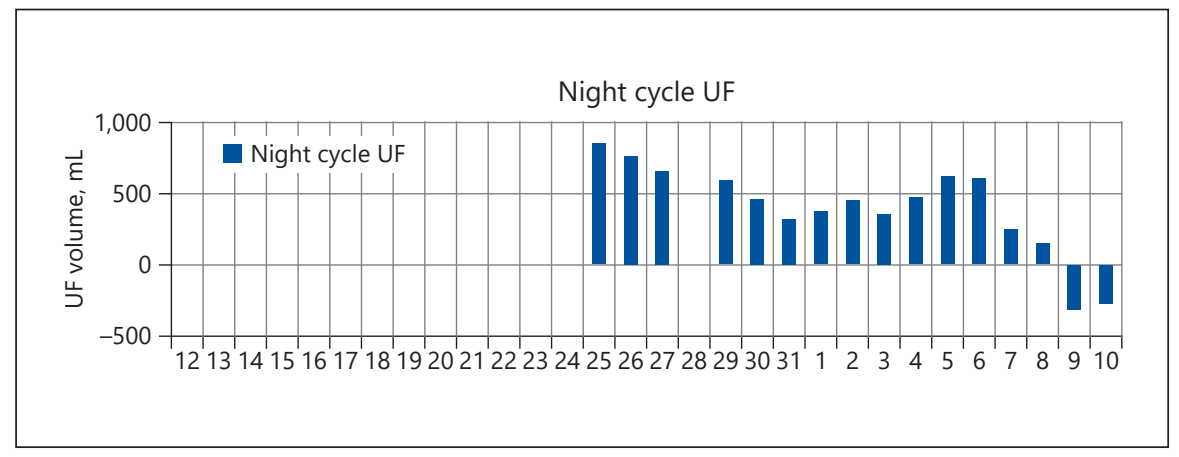

Fig. 3. Sudden change in UF profile from a patient who developed a retroperitoneal leak. UF, ultrafiltration.

propriately placed and there was no outward evidence of a leak on physical examination. However, the patient had returned to PD after a nephrectomy, and a CT scan demonstrated a retroperitoneal leak leading to suspension from PD and a period of hemodialysis. The sudden change in UF noted on sequential monitoring data lead to prompt investigation and diagnosis of a retroperitoneal leak.

\section{Hospital and Home Visits}

Clearly there are potential efficiencies in the use of remote technologies that can reduce the requirement for direct patient contact - whether it is in terms of the patient attending the hospital for review or the nursing team visiting the patient at home. This would result in reduced travel costs and time for patients and healthcare professionals, as well as easing the burden on acute medical care services. Prominent among this is the ability to adjust the prescription remotely, providing the opportunity for responsive changes to be made in a timely fashion. Examples include the need to increase dialysate volumes or dwell times, or make adjustments to the tidal prescriptions. It is important that such adjustments are made in discussion with the patient. As part of this, it is relevant to know which aspects of RM contribute positively to patient experience and wellbeing, and which are less helpful. By reviewing treatment data such as cycler alarms, UF values, BP, and weights more frequently, there is the potential to reduce hospitalizations. However, the evidence for this is so far not consistent since routine monitoring of weight was associated with lower rates, but an increase was seen where routine BP data was monitored [18, 19]. This area requires further study to determine triggers for intervention and admission. 
Table 2. Patient quotes from the Sheffield clinic about their experience of remote patient management

Responsiveness of care

The nurses were already thinking about solutions to my problems by the time I called

Confidence in care

It feels like having someone coming around and checking up on her every day. It

was difficult before because we live very far away so now it saves them from having to come out to see us

It made me feel more secure knowing that they had the information available to them, and so added an additional layer of monitoring'

Privacy

I'm not worried about privacy. From my perspective, the more information they can get, the better it is

\section{Patient Satisfaction and Adherence to Therapy}

Documentation of their observations and vital signs is challenging for patients and adds to the burden of the therapy, particularly in those who have language, educational or cognitive barriers. However, in a cohort study of 300 patients at 10 centers, the use of telehealth increased patients' perceived autonomy and confidence regarding PD treatments as well as satisfaction with care provided over the phone and via remote biometric monitoring. Participants were less likely to report lower health status, and there was high satisfaction with the telehealth interface [20]. Non-adherence has been associated with poorer outcomes - such as TF, inadequate dialysis, peritonitis, and hospitalization [21] and may be a marker of "burn-out." There is, therefore, potential value in measuring the level of adherence that patients have with their home dialysis therapies - for example, in relation to missed or shortened treatments. Understanding the frank reality of the impact of missed therapy can be helpful to patients, however, this information needs to be presented sensitively, with a clear explanation of the benefits of supervised adherence as part of the healthcare partnership. Table 2 includes a number of patient comments from our clinic showcasing their experience in the areas of responsiveness of care, confidence in care, and privacy (Table 2).

\section{Data Safety and Reliability}

Part of the responsibility of organizations and doctors is to ensure patient data are stored and handled safely and in a manner to reduce loss or theft. A recent attack on SingHealth showcased the potential vulnerabilities of digitalized data [22]. These data breaches create mistrust and understandably, patients are concerned about how their information is protected. This is a significant chal- 
lenge for healthcare organizations in the modern digital age and robust vigilance is necessary to ensure safe storage. Patients need to be provided with information on how their data is processed and used to build confidence. The reliability of data transmission is also key and the team have to be vigilant to instances of data not being captured due to connectivity problems, which results in incomplete data and possible misinterpretation leading to inappropriate intervention.

\section{Conclusion}

It is anticipated that remote technologies will improve clinical outcomes through earlier recognition and correction of problems [10]. For patients on home dialysis, telemedicine provides a range of opportunities - including more responsive support, tracking relevant data, supporting the scheduling of supplies, an educational resource, as well as providing a means to enhance patient confidence and reduce anxiety. Ultimately a key question is whether its use will help to reduce rates of TF. It is important that evidence is developed of its use through well-designed research studies such as PD Outcomes and Practice Patterns Study [14].

\section{References}

1 Vegesna A, Tran M, Angelaccio M, Arcona S: Remote patient monitoring via non-invasive digital technologies: a systematic review. Telemed J E Health 2017;23:3-17.

2 Hanlon P, Daines L, Campbell C, McKinstry B, Weller D, Pinnock H: Telehealth interventions to support self-management of long-term conditions: a systematic metareview of diabetes, heart failure, asthma, chronic obstructive pulmonary disease, and cancer. J Med Internet Res 2017; 19:e172.

3 de Jong MJ, van der Meulen-de Jong AE, Romberg-Camps MJ, Becx MC, Maljaars JP, Cilissen $\mathrm{M}$, et al: Telemedicine for management of inflammatory bowel disease (myIBDcoach): a pragmatic, multicentre, randomised controlled trial. Lancet 2017;390:959-968.

4 Hibbard J, Gilburt H: Supporting People to Manage Their Health. An Introduction to Patient Activation. The King's Fund, 2014.

5 National Institute of Health and Care Excellence: Renal Replacement Therapy and Conservative Management, 2018.
6 Patient Reported Experience of Kidney Care in England and Wales 2017. UK Renal Registry, Kidney Care UK, 2018.

7 Rosner MH, Lew SQ, Conway P, Ehrlich J, Jarrin R, Patel UD, et al: Perspectives from the kidney health initiative on advancing technologies to facilitate remote monitoring of patient self-care in RRT. Clin J Am Soc Nephrol 2017;12:1900-1909.

8 Nayak KS, Ronco C, Karopadi AN, Rosner MH: Telemedicine and remote monitoring: supporting the patient on peritoneal dialysis. Perit Dial Int 2016;36:362-366.

9 Lew SQ, Sikka N: Are patients prepared to use telemedicine in home peritoneal dialysis programs? Perit Dial Int 2013;33:714-715.

10 Wallace EL, Rosner MH, Alscher MD, Schmitt CP, Jain A, Tentori F, et al: Remote patient management for home dialysis patients. Kidney Int Rep 2017;2:1009-1017.

11 Jennette C, Derebail V, Baldwin J, Cameron S: Renal replacement therapy and barriers to choice: using a mixed methods approach to explore the patient's perspective. J Nephrol Soc Work 2009; 32:15-26. 
12 Ziolkowski S, Liebman S: A qualitative assessment of mismatch between dialysis modality selection and initiation. Perit Dial Int 2016;36:463466.

13 Campbell DJ, Craig JC, Mudge DW, Brown FG, Wong G, Tong A: Patients' perspectives on the prevention and treatment of peritonitis in peritoneal dialysis: a semi-structured interview study. Perit Dial Int 2016;36:631-639.

14 Perl J, Davies SJ, Lambie M, Pisoni RL, McCullough K, Johnson DW, et al: The peritoneal dialysis outcomes and practice patterns study (PDOPPS): unifying efforts to inform practice and improve global outcomes in peritoneal dialysis. Perit Dial Int 2016;36:297-307.

15 See EJ, Johnson DW, Hawley CM, Pascoe EM, Badve SV, Boudville N, et al: Risk predictors and causes of technique failure within the first year of peritoneal dialysis: an Australia and New Zealand dialysis and transplant registry (ANZDATA) study. Am J Kidney Dis 2018;72:188-197.

16 Drepper VJ, Martin PY, Chopard CS, Sloand JA: Remote patient management in automated peritoneal dialysis: a promising new tool. Perit Dial Int 2018;38:76-78.
17 Lew SQ, Sikka N, Thompson C, Cherian T, Magnus $\mathrm{M}$ : Adoption of telehealth: remote biometric monitoring among peritoneal dialysis patients in the united states. Perit Dial Int 2017;37:576-578.

18 Lew SQ, Sikka N, Thompson C, Magnus M: Impact of remote biometric monitoring on cost and hospitalization outcomes in peritoneal dialysis. J Telemed Telecare 2018:1357633X18784417.

19 Dey V, Jones A, Spalding EM: Telehealth: acceptability, clinical interventions and quality of life in peritoneal dialysis. SAGE Open Med 2016;4: 2050312116670188.

20 Magnus M, Sikka N, Cherian T, Lew SQ: Satisfaction and improvements in peritoneal dialysis outcomes associated with telehealth. Appl Clin Inform 2017;8:214-225.

21 Bernardini J, Piraino B: Compliance in CAPD and CCPD patients as measured by supply inventories during home visits. Am J Kidney Dis 1998;31: 101-107.

22 Vincent J: 1.5 Million Affected by Hack Targeting Singapore's Health Data 2018 https://www.theverge.com/2018/7/20/17594578/singapore-healthdata-hack-sing-health-prime-minister-lee-targeted.
Martin Wilkie

Sheffield Kidney Institute

Sheffield Teaching Hospital NHS Foundation Trust

Herries Road

Sheffield S5 7AU (UK)

E-Mail martin.wilkie@sth.nhs.uk 